\title{
Nuclear medicine and oncology
}

\author{
Peter Lind, Hans-Jürgen Gallowitsch
}

Published online: 26 October 2012

(C) Springer-Verlag Wien 2012

Medical treatment of malignant tumors has changed in the last years from nonspecific systemic chemotherapy to tumor-specific, patient-tailored therapy due to the introduction of tumor-specific pharmaceuticals interfering with intracellular biochemical pathways, affecting proliferation, signal transduction, RNA-replication, and apoptosis. Due to the fact that nuclear medicine (NM) procedures can image many of these metabolic and biochemical processes, it does not only play an incremental role in patient-tailored specific diagnostics but also in therapeutic procedures. Since the introduction of commercially available PET-CT scanners in the last 2 decades and the evolution of new, more or less specific tumor-seeking agents, it is not only possible to better detect malignant tumors and giving prognostic information, but also rendering additional information about the biochemical behavior of each tumor. Due to the ability to image receptor availability and density, glucose or amino acid metabolism, tumor hypoxia and membrane proliferation, PET has a significant impact on the therapeutic management.

In differentiated thyroid cancer (DTC), NM traditionally covers all aspects in patient management from primary diagnosis to nonsurgical therapy and follow-up, keeping patient's management in one institution. In an overview by $S$. Kohlfürst, all these diagnostic and therapeutic tools in the context of DTC are discussed. Ultrasound and ultrasonography-guided fine-needle aspiration biopsy together with scintigraphy in some cases are the most adequate tools for primary diagnosis. In the followup of DTC, thyroglobulin performs as a tumor marker, the major follow-up instrument, supplemented by neck ultrasound and diagnostic ${ }^{131}$ Iodine whole body scan. The introduction of single-photon emission computed tomography (SPECT/CT) allows a better discrimination

\footnotetext{
Univ.Prof.Dr. H.-J. Gallowitsch, MD $(\bowtie) \cdot$ Univ.Doz.Dr. P. Lind Department of Nuclear Medicine and Endocrinology, PET/CT Centre, Klinikum Klagenfurt am Wörthersee, Feschnigstrasse 11, 9020 Klagenfurt, Austria e-mail: hans.gallowitsch@kabeg.at
}

of thyroid remnants and cervical lymph node metastases and allows a better therapeutic management. Positron emission tomography (PET/CT) with ${ }^{18} \mathrm{~F}$-fluorodeoxyglucose (FDG) is also capable to demonstrate iodinenegative metastases which are responsible for a worse prognosis in these cases. With the development of ${ }^{68} \mathrm{Ga}$ somatostatin receptor (SSTR) PET-CT, the NM spectrum has further increased. After surgical thyroidectomy, remnant ablation with ${ }^{131}$ Iodine is routineously performed in a NM therapy-ward as a standard procedure in most of the cases with regard to histology. In case of iodine-positive metastases, repeated therapies can be performed in order to reduce tumor burden.

Neuroendocrine tumors (NET) are rare and usually slowly growing malignant neoplasms. In the article by Sorschag et al. in this edition, he demonstrates that NM is feasible to visualize the presence of molecular biomarkers in these tumors, particularly the overexpression of SSTR with high diagnostic accuracy. Traditionally, this was performed by ${ }^{111}$ In-labeled SSTR-analogues, recently replaced by ${ }^{99 \mathrm{~m}} \mathrm{Tc}$-labeled tracers. The last development is SSTR-PET-CT using ${ }^{68} \mathrm{Ga}$-labeled synthetical SSTRanalogues such as DOTA-NOC, DOTA-TOC, or DOTATATE, which soon established as the gold standard of functional imaging in the workup of NET. These imaging agents allow to select patients with inoperable or metastasized tumors showing sufficient uptake for dosimetry and peptide receptor radionuclide therapy (PRRT).

Gastrointestinal stromal tumors (GISTs) are also rare neoplasms, accounting for less than $3 \%$ of all gastrointestinal malignancies. Molecular-targeted treatment regimes such as tyrosine kinase inhibitors, (e.g., imatimib or sunatinib) have revolutionized the management of patients with irresectable GISTs or metastatic disease. ${ }^{18} \mathrm{~F}-\mathrm{FDG}$ PET-CT is able to display the increased glucose metabolism in malignant GISTs and is a useful tool to evaluate treatment response, possible resistance to imatimib and for early detection of tumor recurrence. The article by $P$. Malle et al. sheds light on the impact of FDG PET and combined FDG PET/CT imaging in this tumor entity. 
In an overview by $M$. Gabriel named "Radionuclide therapy beyond Radioiodine", four different radionuclide therapies using $\beta$-emitters with different ligands are more closely presented. The PRRT using ${ }^{90} \mathrm{Y}$ or ${ }^{177} \mathrm{Lu}-$ labeled SSTR ligands is performed in patients with neuroendocrine tumors when previous SSTR-scintigraphy or PET-CT demonstrated an extensive tumor load with overexpression of SSTR. Radioimmunotherapy with ${ }^{90} \mathrm{Y}$ radio-labeled antibodies against CD20-positive cells is used in the treatment of therapy-resistant, indolent nonHodgkin lymphoma with high remission rates and promising prognostical benefit. Using selective intraarterial radio therapy (SIRT) ${ }^{90} \mathrm{Y}$-labeled particles can be administered in primary or secondary liver tumors. Bone-seeking radio-labeled tracers such as ${ }^{153} \mathrm{Sm}$ EDTMP or ${ }^{89} \mathrm{Sr}$ can be injected to patients with painful bone metastases for pain palliation when conventional treatment regiments fail.
In the last 2 decades, NM has made a great step into the oncological field-not only in the diagnosis of several tumors, but was also implemented in several multimodal therapeutic concepts in oncology which led to the new affiliation "Nuclear Oncology". The introduction of FDGPET into clinical routine was the initial trigger with the subsequent development of several radiopharmaceuticals used for individualized tumor- and patient-tailored diagnostic and therapeutic procedures.

We are pleased to cover the topic "Nuclear Medicine and Oncology" in this issue of Wiener Medizinische Wochenschrift. Special thanks to the editor of the journal Peter Mikosch, who was years ago member of our NM family in Klagenfurt and who is responsible that we have the honor to organize this issue.

\section{Conflict of interest}

For both authors, there is no conflict of interest. 